\title{
A Less Invasive Approach to Rheology Measurements
}

\author{
Researchers have demonstrated a method of probing a soft material's \\ properties that could allow them to capture those properties more \\ accurately and for smaller systems than current methods. \\ By Katherine Wright
}

$\mathrm{M}$ easuring the material properties of a cell is tricky: Researchers typically make such measurements by inserting beads into the cell and then inferring its mechanical properties from the way those beads move. But the beads can change the properties of their immediate environment, causing erroneous measurements. Furthermore, the beads are generally too big to insert inside subcellular systems, such as the nucleus and the cell cortex. A new technique from Christoph Schmidt at Duke University, North Carolina, and colleagues could offer a less disruptive alternative [1]. The researchers show that they can perform localized rheological measurements of soft materials using carbon-nanotube filaments; objects that are long enough to track using a microscope but narrow enough to insert into subcellular systems without significantly perturbing their structures.

In their demonstration, the team mixed single-walled carbon

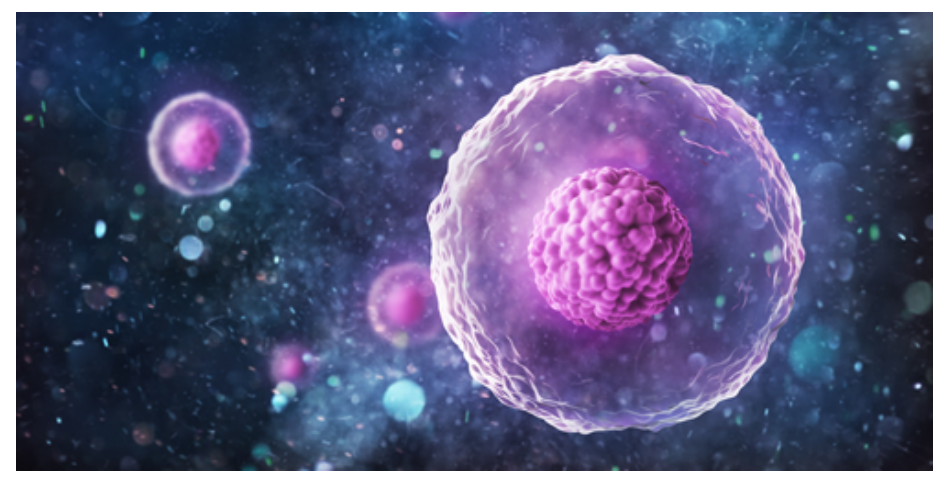

Credit: vipman4/stock.adobe.com nanotubes into various polymer-containing fluids, then sandwiched the mixtures between two glass slides. Using a fluorescence microscope, they observed the fluorescence signals of the nanotubes and used those signals to precisely track how the shapes of the nanotubes fluctuated in response to thermally induced motions of the fluids. The bending stiffness of carbon nanotubes is well defined, so the team was able to use that information to deduce the fluids' mechanical properties.

Now that they know that the method works, Schmidt and colleagues plan to try to perform these measurements using actin filaments-natural carbon-nanotube-like polymers that already exist in cells. Using native filaments would avoid the need to introduce foreign objects into the cell, making such measurements much more reliable.

Katherine Wright is the Deputy Editor of Physics.

\section{REFERENCES}

1. K. Nishi et al., "Multiscale microrheology using fluctuating filaments as stealth probes," Phys. Rev. Lett. 127, 158001 (2021). 